\title{
Сравнительная оценка качества жизни пациентов после холецистэктомии в зависимости от вида доступа и характера течения заболевания
}

\author{
В.И. Давыдкин ${ }^{1}$, Р.И. Федулов ${ }^{1}$, Ю.А. Соболев ${ }^{2}$, А.И. Беляева ${ }^{2}$ \\ ${ }^{1}$ Национальный исследовательский Мордовский государственный университет, \\ Россия, 430005, г. Саранск, ул. Большевистская, 68 \\ ${ }^{2}$ Оренбургский государственный медицинский университет, \\ Россия, 460000, г. Оренбург, ул. Советская / ул. М. Горького / пер. Дмитриевский, 6/45/7 \\ E-mail: v-dav@mail.ru, fedulovruslan@yandex.ru, y_sobolev@mail.ru, sasha1115@inbox.ru
}

\begin{abstract}
Аннотация. Распространенность желчнокаменной болезни среди трудоспособного населения составляет 10-20\%. Холецистэктомия (ХЭ) занимает первое место по частоте среди хирургических вмешательств. Для оценки эффективности лечения важным является анализ качества жизни (КЖ) оперированных и не оперированных больных. Целью работы является оценка показателей качества жизни у больных холелитиазом через 6 месяцев после ХЭ, выполненных на базе трёх медицинских учреждений России. Группы пациентов были однородны по своему составу. КЖ оценивалось с помощью опросника Gallstone Impact Checklist, который включает в себя 4 клинических цифровых шкалы, оценивающих КЖ (шкалы боли, диспепсии, эмоций, питания). Сравнение проводилось между группами в зависимости от характера оперативного доступа (лапароскопический, мини-доступ) и течения заболевания (симптомный и бессимптомный варианты). Основным фактором снижения качества жизни после операции является сохранение болевого синдрома. После ЛХЭ болевой синдром стал менее выраженным, однако иные показатели качества жизни ухудшились. Эмоционально-волевая сфера жизнедеятельности пациентов страдает меньше после ХЭ из «мини-доступа». После операции у пациентов с симптомным течением болезни наблюдается улучшение показателей КЖ по сравнению с группой с бессимптомным течением.
\end{abstract}

Ключевые слова: cholelithiasis, хронический калькулезный холецистит, лапароскопическая холецистэктомия, холецистэктомия из мини-доступа, результаты операции, качество жизни.

Для цитирования: Давыдкин В.И., Федулов Р.И., Соболев Ю.А., Беляева А.И. 2020. Сравнительная оценка качества жизни пациентов после холецистэктомии в зависимости от вида доступа и характера течения заболевания. Актуальные проблемы медицины, 43 (3): 441-454. DOI: $10.18413 / 2687-0940-2020-43-3-441-454$

\section{Comparative assessment of the quality of life of patients after cholecystectomy depending on the type of access and the nature of the disease course}

\author{
Vasily I. Davydkin ${ }^{1}$, Ruslan I. Fedulov ${ }^{1}$, Yuri A. Sobolev ${ }^{2}$, Alexandra I. Belyaeva ${ }^{2}$ \\ ${ }^{1}$ National Research Mordovia State University, \\ 68 Bolshevistskaia St., Saransk, 430005, Russia \\ ${ }^{2}$ Orenburg State Medical University, \\ 6/45/7, Sovetskaja St. / M. Gorkogo St. / Dmitrievsky Lane, 460000, Orenburg, Russia \\ E-mail: v-dav@mail.ru, fedulovruslan@yandex.ru,y_sobolev@mail.ru, sasha1115@inbox.ru
}

Abstract. The prevalence of cholelithiasis among the working population ranges from $10 \%$ to $20 \%$. Cholecystectomy (CE) ranks first in terms of frequency among surgical operations. To assess the 
effectiveness of treatment, it is important to analyze the quality of life (QOL) of operated and nonoperated patients. The aim is to assess the quality of life indicators in patients with cholelithiasis 6 months after cholecystectomy. The patient groups were homogeneous in composition. The operations were performed on the basis of three medical clinics in Russia. QoL was assessed using the Gallstone Impact Checklist, which includes 4 clinical digital scales for evaluating QoL (pain, dyspepsia, emotion, nutrition). The comparison was carried out between groups depending on the surgical approach (laparoscopic, mini-access) and the clinic of the disease (symptomatic and asymptomatic variants). The main factor in reducing the quality of life after surgery is the persistence of pain. After LCE, the pain syndrome became less pronounced, but other indicators of the quality of life worsened. The emotional-volitional sphere of life of patients decreases less after CE from the «mini-access». After surgery, patients with a symptomatic course of the disease have an improvement in QOL indicators compared to the group with an asymptomatic course of the disease.

Keywords: cholelithis, chronic calculous cholecystitis, laparoscopic cholecystectomy, mini-access cholecystectomy, results of surgery, quality of life.

For citation: Davydkin V.I., Fedulov R.I., Sobolev Yu.A., Belyaeva A.I. 2020. Comparative assessment of the quality of life of patients after cholecystectomy depending on the type of access and the nature of the disease course. Challenges in Modern Medicine, 43 (3): 441-454 (in Russian). DOI: 10.18413/2687-0940-2020-43-3-441-454

\section{Введение}

Желчнокаменная болезнь (ЖКБ) является одной из самых распространенных заболеваний гепатобилиарной системы. Она встречается у 10-20 \% лиц трудоспособного населения [Lammert et al., 2016]. Холецистэктомия (ХЭ) занимает первое место по частоте среди хирургических вмешательств в неотложной и плановой хирургии [Дорофеенков, 2006]. При этом пациенты с осложненной ЖКБ связывают с операцией купирование симптомов болезни, которые снижают качество жизни [Parkin et al., 2017; Alexander et al., 2019]. Однако это происходит не всегда, а пациенты вынуждены вновь обращаться за медицинской помощью [Lamberts et al., 2016]. Хронический калькулезный холецистит в большинстве случаев протекает с многократными приступами желчных колик, но иногда заболевание протекает бессимптомно [Ивашкин, 2016].

Оценка качества жизни (КЖ) в современной медицине играет важную роль, а также может предоставить комплексную оценку состояния здоровья пациента [Koishibayeva, 2017] и помочь пациентам и врачам в принятии клинических решений [Terwee et al., 2012]. Для оценки эффективности хирургического лечения холелитиаза важным является проведение сравнительного анализа качества жизни (КЖ) групп оперированных и не оперированных больных [Zapf et al., 2013; Howie et al., 2017; McLean et. al., 2017], экстренной или плановой ХЭ [Yu et al., 2018], поиск наиболее оптимальных методов и схем лечения ЖКБ: однопортовая [Reibetanz J. et al., 2013] или классическая ЛХЭ [Ito et al., 2019]. Рядом авторов показано, что КЖ улучшается после плановой ЛХЭ в краткосрочной [Alexander et al., 2019] или долгосрочной перспективе [Agathis et al., 2020]. Другими авторами, выявлены различия в результатах хирургического лечения при симптомном и бессимптомном течении холелитиза [Макарова и др., 2017; Wanjura, Sandblom, 2016; Lamberts et al., 2016; Howie et al., 2017].

Наиболее распространенным способом оценки качества жизни пациентов является использование опросника MOS SF-36 [Митушева и др., 2015, Howie et al., 2017], PROM [Casanas et al., 2011; McLean et. al., 2017; Alexander et al., 2019] или визуальной аналоговой шкалы (VAS) [Lamberts et al., 2016], они не имеют специфичности в оценке КЖ пациентов с ЖКБ и после ХЭ. Другой опросник Gastrointestinal Quality of Life In$\operatorname{dex}(G I Q L I)$ предназначен для оценки КЖ пациентов с патологией желудочнокишечного тракта [Chen et al. 2006; Sandblom et al., 2009; Abd Ellatif et al., 2013; Lam- 
berts et al., 2015, 2016]. Увеличение индекса QOL коррелирует со сроком после операции, выполненной в плановом или экстренном порядке, доступом, возрастом и полом [Lamberts, 2015, Koishibayeva, 2017, 2020].

Оптимальным для больных, страдающих заболеваниями желчевыводящих путей, является опросник Gallstone Impact Checklist (GIC) [Russell et.al., 1996; Ainslie, 2003; Kirk et al., 2011; Wanjura et al., 2014, 2016].

Цель: провести оценку качества жизни больных холелитиазом и спустя 6 месяцев после лапароскопической ХЭ из мини-доступа.

\section{Объекты и методы исследования}

Проспективное исследование подразумевало анализ качества жизни пациентов, оперированных в 2019 г. в трёх медицинских учреждениях г. Саранска (Республиканская клиническая больница № 4, Мордовская республиканская центральная клиническая больница) и г. Оренбурга (ООО «Клиника промышленной медицины»). Все пациенты были проинформированы о цели и сущности исследования и от каждого было получено информированное согласие на исследование. Исследование было одобрено локальным этическим комитетом Национального исследовательского Мордовского государственного университета.

Методом сплошной выборки сформированы группы пациентов с симптомным холелитиазом (СГ) и латентным течением заболевания (ЛГ), а также группы пациентов после лапароскопической холецистэктомии (ЛХЭ) и ХЭ из мини-доступа (ХЭМД). Все группы сопоставимы по возрасту и полу.

К критериям включения пациентов в исследование отнесли возраст от 18 до 60 лет; физическую полноценность (в т. ч. отсутствие операций на органах гепатодуоденальной зоны); отсутствие заболеваний, подлежащих наблюдению в психоневрологическом и наркологическом диспансерах; наличие информированного согласия на участие в исследовании пациента, в сроки 165-195 дней после операции.

Критерии исключения включали возраст старше 60 лет; пациенты с коморбидной и тяжелой сопутствующей патологией; наличие операций на органах гепатодуоденальной зоны; механическую желтуху; осложнения ХЭ, опухоли брюшной полости; а также предоставление анкет ранее или позднее 165-195 дней после выполненной ХЭ.

За симптомное течение принимали наличие приступов желчной колики в течение 1 года, за латентное течение - отсутствие колики в течение 1 года, предшествующего ХЭ.

Качество жизни определяли путем анкетирования пациентов с использованием валидизированного специфического для больных холелитиазом опросника Gallstone Impact Checklist (GIC) [Russell et.al., 1996]. GIC включает 4 клинических цифровых шкалы, оценивающих качество жизни (шкалы боли, диспепсии, эмоций, питания). Более высокий балл соответствует большей выраженности проблемы и худшему качеству жизни. Шкала боли характеризует интенсивность болевого синдрома. Выраженность диспепсического синдрома отражает шкала диспепсии. Шкала эмоций характеризует влияние заболевания на эмоционально-волевую сферу жизнедеятельности человека. Необходимость соблюдение диеты, ограничение в потребление пищи, необходимость приема ферментативных лекарственных средств отражает шкала питания [Григорьева, 2004]. Пациенты до операции заполняли анкеты в присутствии одного наблюдателя из группы исследователей, после операции участники исследования анкету заполняли без наблюдателей.

Результаты рассчитывали в виде среднего рассчитанного показателя (M), стандартного отклонения (m), 95 \% доверительного интервала (ДИ) и р-критерия. Статистическая обработка была проведена с применением пакетов программ Statistica 12.0 и 
включала создание базы данных, автоматизированную проверку качества подготовки информации и статистический анализ.

\section{Результаты и их обсуждение}

Было обследовано 82 пациента, средний возраст составил 56,2 $\pm 10,3$ лет (в том числе мужчин - 57,2 \pm 10,2 года, женщин - 55,6 \pm 10,3 года). Длительность заболевания до операции составила 8,7 $\pm 3,5$ года. Средний срок после ХЭ составил $180 \pm 12,5$ дней. Сравнительная характеристика групп представлена в табл. 1.

Таблица 1

Table 1

Сравнительная характеристика групп пациентов в зависимости от места исследования Comparative characteristics of patient groups depending on the study site

\begin{tabular}{|l|c|c|c|}
\hline \multicolumn{1}{|c|}{ Город } & $\begin{array}{c}\text { г. Саранск } \\
\mathrm{n}=45\end{array}$ & $\begin{array}{c}\text { г. Оренбург } \\
\mathrm{n}=37\end{array}$ & p-критерий \\
\hline Средний возраст, лет & $47 \pm 10,3$ & $52 \pm 8,4$ & $\mathrm{p}>0,05$ \\
\hline Средний возраст мужчин, лет & $47,2 \pm 10,2$ & $51,1 \pm 8,1$ & $\mathrm{p}>0,05$ \\
\hline Средний возраст женщин, лет & $46,6 \pm 10,3$ & $53,6 \pm 8,7$ & $\mathrm{p}>0,05$ \\
\hline Средняя давность заболевания, лет & $8,7 \pm 3,5$ & $7,9 \pm 3,1$ & $\mathrm{p}>0,05$ \\
\hline Средний срок после холецистэктомии, дней & $180 \pm 12,5$ & $180 \pm 12,5$ & $\mathrm{p}>0,05$ \\
\hline
\end{tabular}

В общей группе пациентов произошло достоверно значимое ухудшение качества жизни по всем показателям, кроме шкалы боли (см. рис. 1), характеризующей интенсивность болевого синдрома (табл. 2).

Таблица 2

Table 2

Качество жизни пациентов до и после операции, в баллах

Patient quality of life before and after surgery

\begin{tabular}{|c|c|c|c|}
\hline Показатели КЖ & До операции & После операции & p-критерий \\
\hline Общий счёт & $\begin{array}{c}75,9 \pm 3,8 \\
\text { (ДИ 74,8-77,0) }\end{array}$ & $\begin{array}{c}85,3 \pm 5,2 \\
\text { (ДИ 83,7-86,9) }\end{array}$ & $\mathrm{p}<0,05$ \\
\hline Счёт шкалы боли & $\begin{array}{c}17,3 \pm 1,8 \\
\text { (ДИ } 16,8-17,8)\end{array}$ & $\begin{array}{c}15,7 \pm 2,7 \\
\text { (ДИ } 14,9-16,5)\end{array}$ & $\mathrm{p}<0,05$ \\
\hline Счёт шкалы диспепсии & $\begin{array}{c}18,2 \pm 1,9 \\
\text { (ДИ } 17,6-18,8)\end{array}$ & $\begin{array}{c}19,3 \pm 2,9 \\
\text { (ДИ } 18,4-20,2)\end{array}$ & $\mathrm{p}<0,05$ \\
\hline Счёт шкалы эмоций & $\begin{array}{c}18,5 \pm 1,5 \\
\text { (ДИ } 18,1-18,9)\end{array}$ & $\begin{array}{c}24,0 \pm 4,2 \\
\text { (ДИ } 22,7-25,3)\end{array}$ & $\mathrm{p}<0,05$ \\
\hline Счёт шкалы питания & $\begin{array}{c}21,9 \pm 1,9 \\
\text { (ДИ } 21,3-22,5)\end{array}$ & $\begin{array}{c}26,2 \pm 2,3 \\
\text { (ДИ 25,5-26,9) }\end{array}$ & $\mathrm{p}<0,05$ \\
\hline
\end{tabular}

Примечание: здесь и далее ДИ - доверительный интервал.

В группе пациентов, перенесших ЛХЭ, средний возраст составил 55,9 \pm 7,1 лет; средняя продолжительность заболевания - 8,2 $\pm 3,4$ года, а в группе пациентов, перенесших ХЭ из мини-доступа, - средний возраст $58,3 \pm 7,0$ лет, средняя продолжительность заболевания $-8,3 \pm 3,4$ лет ( $>0.05)$. Различия в группах были недостоверными.

Качество жизни пациентов до операции в группе с ЛХЭ и с ХЭ из мини-доступа практически было идентичным (рис. 2). 


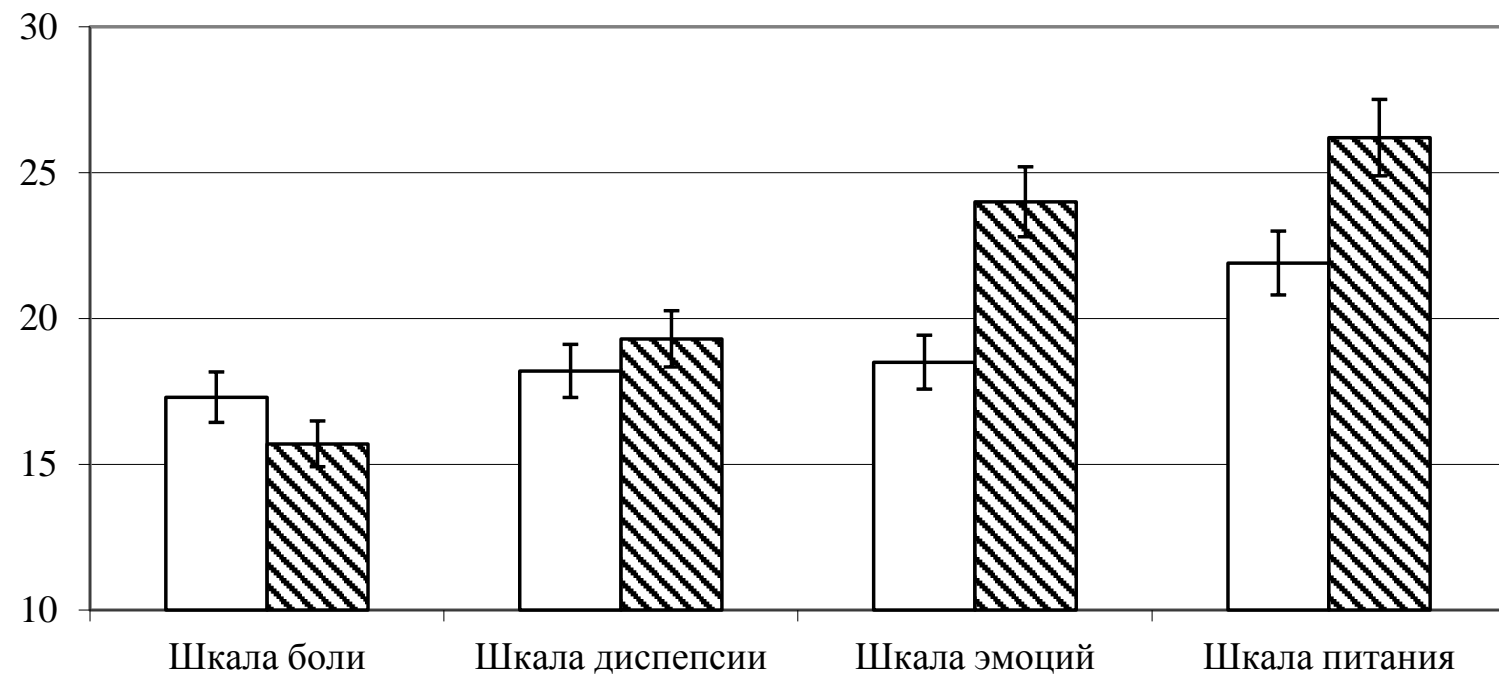

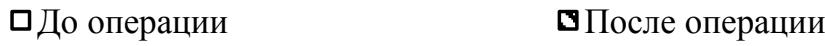

Рис. 1. Сравнение интенсивности болевого синдрома до и после операции

Fig. 1. Comparison of pain intensity before and after surgery

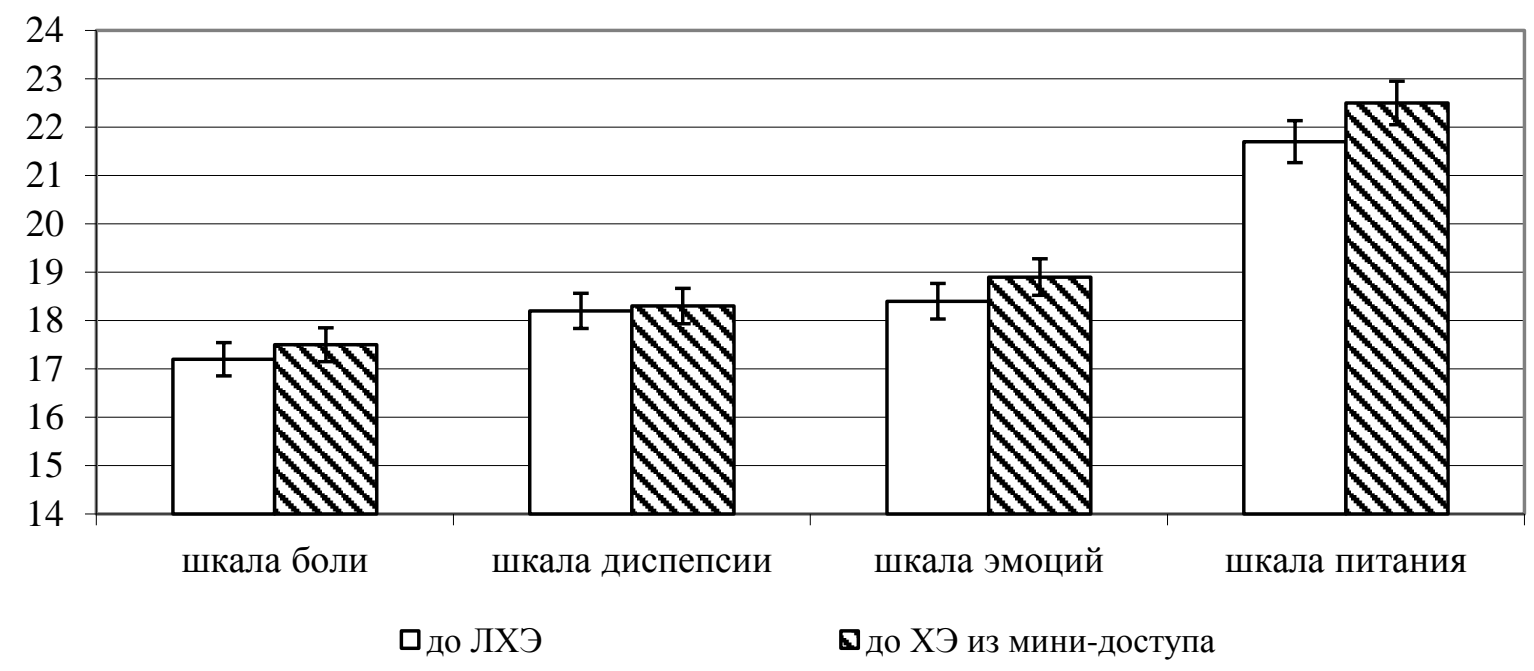

Рис. 2. Качество жизни пациентов до операции в группе с ЛХЭ и с ХЭ из мини-доступа

Fig. 2. Quality of life of patients before surgery in the group with LCE and CE from mini-access

После ЛХЭ отмечено снижение показателей КЖ по шкале общего счета, диспепсии, эмоций и питания, однако отмечено улучшение качества жизни по шкале боли (табл. 3).

Таблица 3

Table 3

Качество жизни пациентов после лапароскопической холецистэктомии

Quality of life of patients after laparoscopic cholecystectomy

\begin{tabular}{|l|c|c|c|}
\hline \multicolumn{1}{|c|}{ Показатели КЖ } & До операции & После операции & p-критерий \\
\hline Общий счёт & $75,4 \pm 3,6$ (ДИ 74,1-76,7) & $85,3 \pm 5,3$ (ДИ 83,4-87,2); & $\mathrm{p}<0,05$ \\
\hline Счёт шкалы боли & $17,2 \pm 2,0$ (ДИ 16,5-17,9) & $14,6 \pm 2,1$ (ДИ 12,5-16,7) & $\mathrm{p}<0,05$ \\
\hline Счёт шкалы диспепсии & $18,2 \pm 2,0$ (ДИ 17,5-18,9) & $19,9 \pm 2,5$ (ДИ 19,0-20,8) & $\mathrm{p}<0,05$ \\
\hline Счёт шкалы эмоций & $18,4 \pm 1,5$ (ДИ 17,9-18,9) & $24,6 \pm 3,9$ (ДИ 20,9-23,5) & $\mathrm{p}<0,05$ \\
\hline Счёт шкалы питания & $21,7 \pm 1,8$ (ДИ 21,1-22,3) & $26,2 \pm 1,8$ (ДИ 25,6-26,8) & $\mathrm{p}<0,05$ \\
\hline
\end{tabular}


В группе пациентов с ХЭ из мини-доступа мало изменилась выраженность болевого и диспепсического синдрома, но отмечено ухудшение показателей шкал общего счета, эмоций и питания (см. табл. 4).

Таблица 4

Table 4

Качество жизни пациентов после холецистэктомии из мини-доступа

Quality of life of patients after cholecystectomy from a mini-access

\begin{tabular}{|c|c|c|c|}
\hline Показатели КЖ & До операции & После операции & p-критерий \\
\hline Общий счёт & $\begin{array}{c}77,2 \pm 4,2 \\
\text { (ДИ 75,9-78,5) }\end{array}$ & $\begin{array}{c}85,1 \pm 4,9 \\
\text { (ДИ } 82,0-88,2) ;\end{array}$ & $\mathrm{p}<0,05$ \\
\hline Счёт шкалы боли & $\begin{array}{c}17,5 \pm 0,9 \\
\text { (ДИ } 16,9-18,1)\end{array}$ & $\begin{array}{c}18,7 \pm 2,0 \\
\text { (ДИ } 17,4-20,0)\end{array}$ & $\mathrm{p}>0,05$ \\
\hline Счёт шкалы диспепсии & $\begin{array}{c}18,3 \pm 1,6 \\
\text { (ДИ } 17,3-19,3)\end{array}$ & $\begin{array}{c}17,7 \pm 3,2 \\
(\text { ДИ } 15,7-19,7)\end{array}$ & $\mathrm{p}>0,05$ \\
\hline Счёт шкалы эмоций & $\begin{array}{c}18,9 \pm 1,6 \\
\text { (ДИ } 17,9-19,9)\end{array}$ & $\begin{array}{c}22,2 \pm 4,5 \\
\text { (ДИ } 19,3-25,1)\end{array}$ & $\mathrm{p}<0,05$ \\
\hline Счёт шкалы питания & $\begin{array}{c}22,5 \pm 2,0 \\
\text { (ДИ } 21,2-23,8)\end{array}$ & $\begin{array}{c}26,5 \pm 3,5 \\
(Д И ~ 24,3-28,8)\end{array}$ & $\mathrm{p}<0,05$ \\
\hline
\end{tabular}

Сравнивая группы пациентов, которым была проведена ЛХЭ и ХЭ из минидоступа, следует отметить, что во второй группе улучшилась эмоционально-волевая сфера жизнедеятельности. В группе пациентов, у которых была проведена ЛХЭ, интенсивность болевого синдрома снизилась. Выбор способа никак не повлиял на выраженность диспепсического синдрома и на необходимость соблюдения диеты, что и оказало влияние на показатель КЖ по шкале общего счета: в обеих группах он стал ниже, но между группами различий не получено (табл. 5).

Таблица 5

Table 5

Сравнительная характеристика групп пациентов в зависимости от тактики оперативного вмешательства

Comparative characteristics of patient groups depending on the tactics of surgical intervention

\begin{tabular}{|c|c|c|c|}
\hline Показатели КЖ & Мини-доступ & $\begin{array}{c}\text { Лапароскопический } \\
\text { доступ }\end{array}$ & $\mathrm{P}$ \\
\hline Общий счёт & $\begin{array}{c}85,1 \pm 4,9 \\
\text { ДИ 82,0-88,2) }\end{array}$ & $\begin{array}{c}85,3 \pm 5,3 \\
\text { (ДИ 83,4-87,2) }\end{array}$ & $\mathrm{p}>0,05$ \\
\hline Шкала боли & $\begin{array}{c}18,7 \pm 2,0 \\
\text { (ДИ } 17,4-20,0)\end{array}$ & $\begin{array}{c}14,6 \pm 2,1 \\
\text { (ДИ } 12,5-16,7)\end{array}$ & $\mathrm{p}<0,05$ \\
\hline Шкала (диспепсии & $\begin{array}{c}17,7 \pm 3,2 \\
(\text { ДИ } 15,7-19,7) \\
\end{array}$ & $\begin{array}{c}19,9 \pm 2,5 \\
(Д И ~ 19,0-20,8) \\
\end{array}$ & $\mathrm{p}=0,05$ \\
\hline Шкала эмоций & $\begin{array}{c}22,2 \pm 4,5 \\
\text { (ДИ 19,3-25,1) }\end{array}$ & $\begin{array}{c}24,6 \pm 3,9 \\
\text { (ДИ 20,9-23,5) }\end{array}$ & $\mathrm{p}<0,05$ \\
\hline Шкала питания & $\begin{array}{c}26,5 \pm 3,5 \\
\text { (ДИ } 24,3-28,8)\end{array}$ & $\begin{array}{c}26,2 \pm 1,8 \\
\text { (ДИ 25,6-26,8) }\end{array}$ & $\mathrm{p}>0,05$ \\
\hline
\end{tabular}

Средний возраст пациентов с симптомным течением заболевания составил $58,5 \pm 7,1$ лет; средняя продолжительность заболевания $-8,3 \pm 3,5$ лет, а в группе с латентным течением заболевания средний возраст составил 55,1 $\pm 7,0$ лет ( $>0.05)$; средняя продолжительность заболевания $-8,3 \pm 3,4$ лет ( $>0.05)$.

В целом после ХЭ у симптомных пациентов отмечено снижение интенсивности болевого синдрома, а выраженность диспепсического синдрома осталась прежней. Однако на этом фоне отмечено снижение КЖ по шкале общего счета, эмоций и питания (табл. 6). 
Характеристика группы симптомных пациентов

Characteristics of the group of symptomatic patients

\begin{tabular}{|c|c|c|c|}
\hline Показатели КЖ & До операции & После операции & p-критерий \\
\hline Общий счёт & $\begin{array}{c}75,9 \pm 3,9 \\
\text { (ДИ 74,7-77,1) }\end{array}$ & $\begin{array}{c}80,9 \pm 3,5 \\
(\text { (ДИ 79,2-82,6) }\end{array}$ & $\mathrm{p}<0,05$ \\
\hline Счёт шкалы боли & $\begin{array}{c}17,1 \pm 1,9 \\
\text { (ДИ } 16,5-17,7)\end{array}$ & $\begin{array}{c}15,9 \pm 3,5 \\
\text { (ДИ } 14,9-16,9) ;\end{array}$ & $\mathrm{p}<0,05$ \\
\hline Счёт шкалы диспепсии & $\begin{array}{c}18,0 \pm 1,7 \\
\text { (ДИ } 17,5-18,5)\end{array}$ & $\begin{array}{c}18,2 \pm 2,4 \\
\text { (ДИ } 17,5-18,9)\end{array}$ & $\mathrm{p}>0,05$ \\
\hline Счёт шкалы эмоций & $\begin{array}{c}18,6 \pm 1,3 \\
\text { (ДИ } 18,2-19,0)\end{array}$ & $\begin{array}{c}21,3 \pm 2,9 \\
(\text { ДИ 20,4-22,2) }\end{array}$ & $\mathrm{p}<0,05$ \\
\hline Счёт шкалы питания & $\begin{array}{c}22,2 \pm 1,8 \\
\text { (ДИ } 21,7-22,7)\end{array}$ & $\begin{array}{c}25,7 \pm 1,9 \\
(\text { (ДИ } 25,1-26,3)\end{array}$ & $\mathrm{p}<0,05$ \\
\hline
\end{tabular}

В группе пациентов с латентным течением холелитиаза через 6 месяцев после ХЭ отмечено ухудшение качества жизни по всем показателям, за исключение шкалы боли (табл. 7).

Характеристика группы пациентов с латентным течением

Characteristics of the group of patients with latent course

\begin{tabular}{|c|c|c|c|}
\hline Показатели КЖ & До операции & После операции & p-критерий \\
\hline Общий счёт & $\begin{array}{c}75,9 \pm 3,9 \\
\text { (ДИ 74,7-77,1) }\end{array}$ & $\begin{array}{c}88,4 \pm 3,7 \\
\text { (ДИ 86,9-89,9) }\end{array}$ & $p<0,05$ \\
\hline Счёт шкалы боли & $\begin{array}{c}17,4 \pm 1,6 \\
\text { (ДИ } 16,7-18,1)\end{array}$ & $\begin{array}{c}15,6 \pm 2,0 \\
\text { (ДИ } 14,8-16,4)\end{array}$ & $\mathrm{p}<0,05$ \\
\hline Счёт шкалы диспепсии & $\begin{array}{c}18,4 \pm 2,0 \\
\text { (ДИ } 17,6-19,2)\end{array}$ & $\begin{array}{c}20,1 \pm 2,9 \\
\text { (ДИ } 18,9-21,3)\end{array}$ & $\mathrm{p}<0,05$ \\
\hline Счёт шкалы эмоций & $\begin{array}{c}18,4 \pm 1,6 \\
\text { (ДИ } 17,8-19,0\end{array}$ & $\begin{array}{c}26,0 \pm 3,8 \\
\text { (ДИ } 24,5-27,5) \\
\end{array}$ & $\mathrm{p}<0,05$ \\
\hline Счёт шкалы питания & $\begin{array}{c}21,7 \pm 1,9 \\
\text { (ДИ 20,9-22,5) }\end{array}$ & $\begin{array}{c}26,7 \pm 2,5 \\
\text { (ДИ } 25,7-27,7)\end{array}$ & $\mathrm{p}<0,05$ \\
\hline
\end{tabular}

Сравнивая же показатели КЖ пациентов в двух группах, отмечаем, что в группе симптомных пациентов они были лучше по всем шкалам (за исключением шкалы боли), чем в группе с латентным течением заболевания. Выраженность болевых ощущений после ХЭ снижалось в обеих группах вне зависимости от течения заболевания (табл. 8).

Таблица 8

Table 8

Сравнительная характеристика групп пациентов в зависимости от течения заболевания Comparative characteristics of patient groups depending on the course of the disease

\begin{tabular}{|l|c|c|c|}
\hline \multicolumn{1}{|c|}{ Показатели КЖ } & Симптомное течение & Бессимптомное течение & p-критерий \\
\hline Общий счёт & $80,9 \pm 3,5$ (ДИ 79,6-82,6) & $88,4 \pm 3,7$ (ДИ 86,9-89,9) & $\mathrm{p}<0,05$ \\
\hline Шкала боли & $15,9 \pm 3,5$ (ДИ 14,9-16,9) & $15,6 \pm 2,0$ (ДИ 14,8-16,4) & $\mathrm{p}>0,05$ \\
\hline Шкала диспепсии & $18,2 \pm 2,4$ (ДИ 17,5-18,9) & $20,1 \pm 2,9$ (ДИ 18,9-21,3) & $\mathrm{p}<0,05$ \\
\hline Шкала эмоций & $21,3 \pm 2,9$ (ДИ 20,4-22,2) & $26,0 \pm 3,8$ (ДИ 24,5-27,5) & $\mathrm{p}<0,05$ \\
\hline Шкала питания & $25,7 \pm 1,9$ (ДИ 25,1-26,3) & $26,7 \pm 2,5$ (ДИ 25,7-27,7) & $\mathrm{p}<0,05$ \\
\hline
\end{tabular}




\section{Заключение}

ХЭ является единственным окончательным лечением для пациентов с холелитиазом, а ЛХЭ является современным золотым стандартом при неосложненном течении ЖКБ. [Куликовский и др., 2012; Aerts, Penninckx, 2003; Gurusamy, 2014]. При осложнённом же течении рассматриваются возможность выполнения как ЛХЭ, так и ХЭ из «мини-доступа» [Ивашкин, 2016]. Однако, несмотря на высокую эффективность в лечении холелитиаза и распространенность в клинической хирургии, ЛХЭ не лишена рисков и ряда недостатков [Gurusamy, 2014].

Очевидно, что основной целью ХЭ является достижение полного здоровья, но это не всегда происходит. По данным большинства исследователей, показатели КЖ после ХЭ в группе пациентов с симптомным течением заболевания были лучше, чем в группе с латентным течением [Lamberts, 2015]. Связано это с ожиданиями пациентов избавиться от рецидивирующего болевого синдрома путем ХЭ. Пациенты же с латентным течением ожидают от ХЭ избавления от симптомов функциональных нарушений, которые снижают качество их жизни [Литвинова, 2009; Agathis et al., 2020].

Сравнивая результаты оценки КЖ после различных способов ХЭ, установлено ухудшение некоторых показателей качества жизни после ЛХЭ. Хотя выраженность болевого синдрома и стала менее выраженной, что объясняется малой травматичностью операции и удалением очага боли, но он не купировался полностью. Снижение же других показателей КЖ, по-видимому, связано с завышенными ожиданиями пациентов от операции [Ильченко, 2012] и, прежде всего, в группе пациентов с неосложненным течением. Известно, что при длительном течении заболевания формируются различные функциональные расстройства, обусловленные сопутствующей ГЭРБ, дискинезией сфинктера Одди, синдромом раздраженного кишечника. ЖКБ часто сочетается с хроническим панкреатитом, дивертикулярной болезнью, симптомы которых после операции пациентами ошибочно относятся к последствиям самой ХЭ [Винник, Серова, 2013; Вахрушев и др., 2015]. К сожалению, ХЭ не может скорригировать функциональные нарушения, что и способствует снижению субъективной оценки КЖ в послеоперационном периоде по сравнению с группой пациентов с преобладанием болевого синдрома.

Эмоционально-волевая сфера жизнедеятельности страдает в меньшей степени после ХЭ из «мини-доступа» в сравнении с ЛХЭ и в меньшей степени приводит к ограничению жизнедеятельности. Схожую тенденцию пациенты отмечают и в сфере питания. Это связано с необходимостью соблюдения ограничений, хотя большинство пациентов мало склонны соблюдать диету [Плотникова и др., 2011], в связи с чем высока частота появления болевого синдрома [Мартынов, 2013].

На исход операции влияет срочность выполнения хирургического вмешательства и возраст. После плановой ХЭ отмечается снижение КЖ как в первый год, так и на протяжении 5 лет послеоперационного периода [Койшибаева и др., 2018] и реже всего за счет сохраняющихся функциональных нарушений органов гепатопанкреатодуоденальной зоны. После же экстренной ХЭ КЖ пациентов, как правило, улучшается в течение первого года после операции, однако через 5 лет показатели КЖ возвращаются на исходные уровни [Митушева и др., 2015]. Возрастные больные требуют более тщательного динамического наблюдения после операции, разработку индивидуальных программ реабилитации на этапах оказания медицинской помощи и диспансерного наблюдения [Мартынов и др., 2013].

\section{Выводы}

1. ЛХЭ и из ХЭ мини-доступа достоверно, но в разной степени снижают качество жизни пациентов. Выбор ЛХЭ необходим с учетом имеющихся функциональных нарушений органов гепатодуоденальной зоны, которые снижают качество жизни после операции. 
2. ЛХЭ является более предпочтительной у пациентов с преобладанием болевого синдрома в структуре жалоб, являющегося ведущим субъективным фактором снижения качества жизни после операции.

3. ХЭ из мини-доступа не снижает качество жизни пациентов с болевым и диспепсическим синдромами, но ведет к улучшению эмоционально-волевой сферы жизнедеятельности.

4. С целью улучшения качества жизни пациентов после ХЭ, эффективности лечебных и реабилитационных мероприятий целесообразно использование в амбулаторном звене опросника GIC. При этом особое внимание должны заслуживать пациенты с исходным латентным течением холелитиаза, а также перенесшие ЛХЭ и в большей степени нуждающиеся в реабилитационных мероприятиях, нежели пациенты после ХЭ из минидоступа и с симптомным течением холелитиаза.

\section{Конфликт интересов}

Авторы заявляют об отсутствии конфликта интересов.

\section{Список литературы}

1. Вахрушев Я.М., Лукашевич А.П., Горбунов А.Ю. 2015. Изучение функционального состояния тонкой кишки при желчнокаменной болезни. Архивъ внутренней медицины. № 5.

2. Винник Ю.С., Серова Е.В. 2013. Значение холецистокинина октапептида в развитии дисфункции сфинктера Одди неорганической этиологии после холецистэктомии у пациентов, оперированных по поводу калькулёзного холецистита. Пермский медицинский журнал, 30 (5): 77-84.

3. Григорьева И.Н. 2004. Валидация опросника по качеству жизни при желчнокаменной болезни. Российский журнал гастроэнтерологии, гепатологии, колопроктологии. 1: 74-78.

4. Дорофеенков М.Е. 2006. Распространенность и особенности клинической картины желчнокаменной болезни у населения Москвы: дис. ... канд. мед. наук: 14.00 .05 / Дорофеенков Михаил Евгеньевич. 130 с.

5. Ивашкин В.Т. 2016. Рекомендации Российской гастроэнтерологической ассоциации по диагностике и лечению желчнокаменной болезни. Клинические рекомендации РЖГГК. 3: 76.

6. Ильченко А.А. 2012. Рекомендации Научного общества гастроэнтерологов России по диагностике и лечению желчнокаменной болезни и краткие комментарии. Concillium medicum. 14 (8): 21-29.

7. Койшибаева Л.М., Тургунов Е.М., Таласбаев М.А., Саханова А.А. 2018. Сравнение качества жизни пациентов до и после холецистэктомии через 6 и 12 месяцев. Вестник НовГУ. $2(108)$.

8. Куликовский В.Ф., Карпачев А.А., Ярош А.Л., Солошенко А.В. 2012. Малоинвазивные методы лечения осложненной желчнокаменной болезни. Современные проблемы науки и образования. 2: 97.

9. Литвинова Н.В., Осипенко М.Ф. 2009. Структура клинических синдромов и прогностические факторы их развития после холецистэктомии у больных с желчнокаменной болезнью. Бюлл. СО РАМН, 29 (3): 105-109.

10. Макарова Ю.В., Литвинова Н.В., Осипенко М.Ф., Волошина Н.Б. 2017. Абдоминальный болевой синдром и качество жизни у пациентов с желчнокаменной болезнью после холецистэктомии в течение 10 лет наблюдения. Тер. Арx., 89 (2): 70-75. doi: 10.17116 / terarkh201789270-75.

11. Мартынов А.А. 2013. Качество жизни больных пожилого и старческого возраста после лапароскопической холецистэктомии. Журнал МедиАль. 3 (8).

12. Мартынов А.А., Пиксин И.Н., Пигачев А.В. 2013. Изучение динамики сердечной и бронхолегочной патологии у больных пожилого возраста, перенесших лапароскопическую холецистэктомию. Вестник Мордовского университета. 1 (2): 188-190.

13. Митушева Э.И., Сайфутдинов Р.Г., Шаймарданов Р.Ш., Бадретдинова А.Р. 2015. Изменения в органах гепатопанкреатобилиарной системы и качество жизни пациентов после холецистэктомии. Казанский мед. журнал. № 3. 
14. Плотникова Е.Ю., Золотухина В.Н., Максимов С.А., Мухарлямов Ф.Ю. 2011. Качество жизни и приверженность к лечению больных хроническим бескаменным холециститом и желчнокаменной болезнью. Вестник Национального медико-хирургического Центра им. Н.И. Пирогова. № 3.

15. Abd Ellatif M.E., Askar W.A., Abbas A.E., Noaman N., Negm A., El-Morsy G., El Nakeeb A., Magdy A., Amin M. 2013. Quality-of-life measures after single-access versus conventional laparoscopic cholecystectomy: a prospective randomized study. Surg. Endosc.; 27: 1896-906. DOI: 10.1007/s00464-012-2625-5.

16. Aerts R., Penninckx F. 2003. The burden of gallstone disease in Europe. Aliment. Pharmacol. Ther.; 18 Suppl. 3: 49-53. DOI: 10.1046/j.0953-0673.2003.01721.

17. Ainslie W.G., Catton J.A., Davides D., Dexter S., Gibson J., Larvin M., McMahon M.J., Moore M., Smith S., Vezakis A. Micropuncture cholecystectomy vs conventional laparoscopic cholecystectomy: a randomized controlled trial. Surg. Endosc. 2003. 17: 766-72. DOI: 10.1007/s00464-002-8568-5/.

18. Alexander H.C., Nguyen C.H., Moore M.R., Bartlett A.S., Hannam J.A., Poole G.H., Alan F., Merry A.F. 2019. Measurement of patient-reported outcomes after laparoscopic cholecystectomy: a systematic review. Surg. Endosc. 33 (7): 2061-2071. doi: 10.1007/s00464-019-06745-7. Epub 2019 Apr 1.

19. Agathis A.Z., Aalberg J.J., Garvey A., Divino C.M. 2020. Assessing long term quality of life in geriatric patients after elective laparoscopic cholecystectomy. Am. J. Surg. 219 (6): 1039-1044. doi: 10.1016/j.amjsurg.2019.08.021.Epub. 2019, Sep 10.

20. Casanas I.C.C., Gibbons E., Fitzpatrick R.A. 2011. Structured review of patient-reported outcome measures for patients undergoing cholecystectomy. In: Health Do, editor. Department of Public Health, University of Oxford: Patient-Reported Outcome Measurement Group, 1-40.

21. Chen T.Y., Landmann M.G., Potter J.C., van Rij A.M. Questionnaire to aid priority and outcomes assessment in gallstone disease. ANZ J. Surg. 2006 Jul; 76 (7): 569-74. doi: 10.1111/j.14452197.2006.03777.x. PMID: 16813620.

22. Ito E., Takai A., Imai Y., Otani H., Onishi Y., Yamamoto Y., Ogawa K., Tohyama T., Fukuhara S., Takada Y. Quality of life after single-incision laparoscopic cholecystectomy: A randomized, clinical trial. Surgery. 2019 Feb; 165 (2): 353-359. doi: 10.1016/j.surg.2018.08.004. Epub 2018 Oct 9. PMID: 30314725.

23. Gurusamy K.S., Davidson B.R. Gallstones. BMJ. 2014. 348: g 2669. DOI: 10.1136/bmj.g2669.

24. Howie M.T., Sandblom G., Österberg J. 2017. The impact of pain frequency, pain localization and perceived cause of pain on quality of life after cholecystectomy. Scand. J. Gastroenterol. 52 (12): 1391-1397. doi: 10.1080 / 00365521.2017.1369564. Epub. 2017, Aug 28.

25. Kirk G., Kennedy R., McKie L., Diamond T., Clements B. 2011. Preoperative symptoms of irritable bowel syndrome predict poor outcome after laparoscopic cholecystectomy. Surg. Endosc. 25 (10): 3379-84. doi: 10.1007/s00464-011-1729-7. Epub. 2011, May 10.

26. Koishibayeva L.M., Turgunov Ye., Sandblom G., Teleuov M., Baimuratova M. 2017. Assessment of the quality of life of patients after cholecystectomy. Georgian Med. News. (270): 30-38.

27. Koishibayeva L.M., Sandblom G., Turgunov Y.M., Koishibayev Z.M. Quality-of-life assessment after cholecystectomy in a population-based cohort from Kazakhstan. Asian. J. Surg. 2020 Sep.; 43 (9): 948-949. doi: 10.1016/j.asjsur.2020.05.016. Epub 2020 Jun 9. PMID: 32532681.

28. Lamberts M.P., Den Oudsten B.L., Gerritsen J.J.G. M., Roukema J.A., Westert G.P., Drenth J.P.H., van Laarhoven C.J.H.M. 2015. Prospective multicentre cohort study of patient-reported outcomes after cholecystectomy for uncomplicated symptomatic cholecystolithiasis. Br. J. Surg. 102 (11): 1402-9. doi: 10.1002/bjs.9887. Epub. 2015, Jul 22.

29. Lamberts M.P., Kievit W., Gerritsen J.J.G.M., Roukema J.A., Westert G.P., Drenth J. P.H., van Laarhoven C.J.H.M. 2016. Episodic abdominal pain characteristics are not associated with clinically relevant improvement of health status after cholecystectomy. J. Gastrointest. Surg. 20 (7): 1350-8. doi: 10.1007/s11605-016-3156-5. Epub. 2016, May 17.

30. Lammert F., Gurusamy K., Ko C.W., Miquel J.F., Méndez-Sánchez N., Portincasa P., van Erpecum K.J., van Laarhoven C.J., Wang D.Q. Gallstones. Nat. Rev. Dis. Primers. 2016 Apr. 28; 2: 16024. doi: 10.1038/nrdp.2016.24. PMID: 27121416.

31. McLean K.A., Sheng Z., O'Neill S., Boyce K., Jones C., Wigmore S.J., Harrison E.M. 2017. The influence of clinical and patient-reported outcomes on post-surgery satisfaction in cholecystectomy patients. World J. Surg. 41 (7): 1752-1761. doi: 10.1007/s00268-017-3917-7. 
32. Parkin E., Stott M., Brockbank J., Galloway S., Welch I., Macdonald A. 2017. Patientreported outcomes for acute gallstone pathology. World J. Surg. 41 (5): 1234-1238. doi: 10.1007/s00268016-3854-x.

33. Reibetanz J., Ickrath P., Hain J., Germer Ch.-T., Krajinovic K. 2013. Single-port laparoscopic cholecystectomy versus standard multiport laparoscopic cholecystectomy: a case-control study comparing the long-term quality of life and body image. Surg. Today. 43 (9): 1025-30. doi: 10.1007/s00595012-0393-4. Epub. 2012, Nov 2.

34. Russell M.L., Preshaw R.M., Brant R.F. 1996. Disease-specific quality of life: the Gallstone Impact Checklist. Clin. Invest. Med. 19 (6): 453-460.

35. Sandblom G., Videhult P., Karlson B.M., Wollert S., Ljungdahl M., Darkahi B., Liljeholm H., Rasmussen I.C. Validation of Gastrointestinal Quality of Life Index in Swedish for assessing the impact of gallstones on health-related quality of life. Value Health. 2009 Jan-Feb; 12 (1): 181-4. doi: 10.1111/j.1524-4733.2008.00396.x. PMID: 19911447.

36. Terwee C.B., Mokkink L.B., Knol D.L., Ostelo R.W., Bouter L.M., de Vet H.C. Rating the methodological quality in systematic reviews of studies on measurement properties: a scoring system for the COSMIN checklist. Qual. Life Res. 2012 May; 21 (4): 651-7. doi: 10.1007/s11136-011-9960-1. Epub 2011 Jul 6. PMID: $21732199 ;$ PMCID: PMC3323819.

37. Wanjura V., Lundstrom P., Osterberg J. 2014. Gastrointestinal quality-oflife after cholecystectomy: indication predicts gastrointestinal symptoms and abdominal pain. World J. Surg. 38: 3075-81.

38. Wanjura V., Sandblom G., Surg. World J. 2016. How do quality-of-life and gastrointestinal symptoms differ between post-cholecystectomy patients and the background population? 40 (1): 81-88. doi: 10.1007/s00268-015-3240-0.

39. Yu H., Ern-Hwei Chan E., Lingam P., Lee J., Wei Liang Woon W., Keem Low J., Shelat V. 2018. Index admission laparoscopic cholecystectomy for acute cholecystitis restores Gastrointestinal Quality of Life Index (GIQLI) score. Ann. Hepatobiliary Pancreat. Surg. 22 (1): 58-65. doi: 10.14701/ahbps.2018.22.1.58. Epub. 2018, Feb 26.

40. Zapf M., Denham W., Barrera E., Butt Z., Carbray J., Wang Ch., Linn J., Ujiki M. 2013 Patient-centered outcomes after laparoscopic cholecystectomy. Surg. Endosc. 27 (12): 4491-8. doi: 10.1007/s00464-013-3095-0. Epub. 2013, Aug 13.

\section{References}

1. Vahrushev Ja.M., Lukashevich A.P., Gorbunov A.Ju. 2015. Izuchenie funkcional'nogo sostojanija tonkoj kishki pri zhelchnokamennoj bolezni [Study of the functional state of the small intestine in cholelithiasis]. Arhiv" vnutrennej mediciny. № 5.

2. Vinnik Ju.S., Serova E.V. 2013. Znachenie holecistokinina oktapeptida v razvitii disfunkcii sfinktera Oddi neorganicheskoj jetiologii posle holecistjektomii u pacientov, operirovannyh po povodu kal'kuljoznogo holecistita [Significance of cholecystokinin octapeptide in the development of sphincter Oddi dysfunction of inorganic etiology after cholecystectomy in patients operated for calculous cholecystitis]. Permskij medicinskij zhurnal, 30 (5): 77-84.

3. Grigor'eva I.N. 2004. Validacija oprosnika po kachestvu zhizni pri zhelchnokamennoj bolezni [Validation of the quality of life questionnaire for cholelithiasis]. Rossijskij zhurnal gastrojenterologii, gepatologii, koloproktologii, 1: 74-78.

4. Dorofeenkov M.E. 2006. Rasprostranennost' i osobennosti klinicheskoj kartiny zhelchnokamennoj bolezni u naselenija Moskvy [Prevalence and features of the clinical picture of cholelithiasis in the population of Moscow]: dis. ... kand. med. nauk: 14.00.05 / Dorofeenkov Mihail Evgen'evich. $130 \mathrm{c}$.

5. Ivashkin V.T. 2016. Rekomendacii Rossijskoj gastrojenterologicheskoj associacii po diagnostike i lecheniju zhelchnokamennoj bolezni [Recommendations of the Russian gastroenterological Association for the diagnosis and treatment of cholelithiasis]. Klinicheskie rekomendacii RZhGGK. 3: 76.

6. Il'chenko A.A. 2012. Rekomendacii Nauchnogo obshhestva gastrojenterologov Rossii po diagnostike i lecheniju zhelchnokamennoj bolezni i kratkie kommentarii [Recommendations of the Scientific society of gastroenterologists of Russia on the diagnosis and treatment of cholelithiasis and brief comments]. Concillium medicum. 14 (8): 21-29.

7. Kojshibaeva L.M., Turgunov E.M., Talasbaev M.A., Sahanova A.A. 2018. Sravnenie kachestva zhizni pacientov do i posle holecistjektomii cherez 6 i 12 mesjacev [Comparison of quality of life of patients before and after cholecystectomy at 6 and 12 months]. Vestnik NovGU. 2 (108). 
8. Kulikovskij V.F., Karpachev A.A., Jarosh A.L., Soloshenko A.V. 2012. Maloinvazivnye metody lechenija oslozhnennoj zhelchnokamennoj bolezni [Minimally invasive methods of treatment of complicated cholelithiasis]. Sovremennye problemy nauki i obrazovanija. 2: 97.

9. Litvinova N.V., Osipenko M.F. 2009. Struktura klinicheskih sindromov i prognosticheskie faktory ih razvitija posle holecistjektomii u bol'nyh s zhelchnokamennoj bolezn'ju [Structure of clinical syndromes and prognostic factors of their development after cholecystectomy in patients with cholelithiasis]. Bjull. SO RAMN, 29 (3): 105-109.

10. Makarova Ju.V., Litvinova N.V., Osipenko M.F., Voloshina N.B. 2017. Abdominal'nyj bolevoj sindrom i kachestvo zhizni u pacientov s zhelchnokamennoj bolezn'ju posle holecistjektomii $\mathrm{v}$ techenie 10 let nabljudenija [Abdominal pain and quality of life in patients with cholelithiasis after cholecystectomy for 10 years of follow-up]. Ter. Arh., 89 (2): 70-75. doi: 10.17116 / terarkh201789270-75.

11. Martynov A.A. 2013. Kachestvo zhizni bol'nyh pozhilogo i starcheskogo vozrasta posle laparoskopicheskoj holecistjektomii [Quality of life of elderly and senile patients after laparoscopic cholecystectomy]. Zhurnal MediAl'. 3 (8).

12. Martynov A.A., Piksin I.N., Pigachev A.V. 2013. Izuchenie dinamiki serdechnoj i bronholegochnoj patologii u bol'nyh pozhilogo vozrasta, perenesshih laparoskopicheskuju holecistjektomiju [Study of the dynamics of cardiac and bronchopulmonary pathology in elderly patients who underwent laparoscopic cholecystectomy]. Vestnik Mordovskogo universiteta. 1 (2): 188-190.

13. Mitusheva Je.I., Sajfutdinov R.G., Shajmardanov R.Sh., Badretdinova A.R. 2015. Izmenenija $\mathrm{v}$ organah gepatopankreatobiliarnoj sistemy i kachestvo zhizni pacientov posle holecistjektomii [Changes in the organs of the hepatopancreatobiliary system and the quality of life of patients after cholecystectomy]. Kazanskij med. zhurnal. № 3.

14. Plotnikova E.Ju., Zolotuhina V.N., Maksimov S.A., Muharljamov F.Ju. 2011. Kachestvo zhizni i priverzhennost' $\mathrm{k}$ lecheniju bol'nyh hronicheskim beskamennym holecistitom i zhelchnokamennoj bolezn'ju [Quality of life and commitment to treatment of patients with chronic stone-free cholecystitis and cholelithiasis]. Vestnik Nacional'nogo mediko-hirurgicheskogo Centra im. N.I. Pirogova. № 3.

15. Abd Ellatif M.E., Askar W.A., Abbas A.E., Noaman N., Negm A., El-Morsy G., El Nakeeb A., Magdy A., Amin M. 2013. Quality-of-life measures after single-access versus conventional laparoscopic cholecystectomy: a prospective randomized study. Surg. Endosc.; 27: 1896-906. DOI: 10.1007/s00464-012-2625-5.

16. Aerts R., Penninckx F. 2003. The burden of gallstone disease in Europe. Aliment. Pharmacol. Ther.; 18 Suppl. 3: 49-53. DOI: 10.1046/j.0953-0673.2003.01721.

17. Ainslie W.G., Catton J.A., Davides D., Dexter S., Gibson J., Larvin M., McMahon M.J., Moore M., Smith S., Vezakis A. Micropuncture cholecystectomy vs conventional laparoscopic cholecystectomy: a randomized controlled trial. Surg. Endosc. 2003. 17: 766-72. DOI: 10.1007/s00464-002-8568-5/.

18. Alexander H.C., Nguyen C.H., Moore M.R., Bartlett A.S., Hannam J.A., Poole G.H., Alan F., Merry A.F. 2019. Measurement of patient-reported outcomes after laparoscopic cholecystectomy: a systematic review. Surg. Endosc. 33 (7): 2061-2071. doi: 10.1007/s00464-019-06745-7. Epub 2019 Apr 1.

19. Agathis A.Z., Aalberg J.J., Garvey A., Divino C.M. 2020. Assessing long term quality of life in geriatric patients after elective laparoscopic cholecystectomy. Am. J. Surg. 219 (6): 1039-1044. doi: 10.1016/j.amjsurg.2019.08.021.Epub. 2019, Sep 10.

20. Casanas I.C.C., Gibbons E., Fitzpatrick R.A. 2011. Structured review of patient-reported outcome measures for patients undergoing cholecystectomy. In: Health Do, editor. Department of Public Health, University of Oxford: Patient-Reported Outcome Measurement Group, 1-40.

21. Chen T.Y., Landmann M.G., Potter J.C., van Rij A.M. Questionnaire to aid priority and outcomes assessment in gallstone disease. ANZ J. Surg. 2006 Jul; 76 (7): 569-74. doi: 10.1111/j.14452197.2006.03777.x. PMID: 16813620.

22. Ito E., Takai A., Imai Y., Otani H., Onishi Y., Yamamoto Y., Ogawa K., Tohyama T., Fukuhara S., Takada Y. Quality of life after single-incision laparoscopic cholecystectomy: A randomized, clinical trial. Surgery. 2019 Feb; 165 (2): 353-359. doi: 10.1016/j.surg.2018.08.004. Epub 2018 Oct 9. PMID: 30314725.

23. Gurusamy K.S., Davidson B.R. Gallstones. BMJ. 2014. 348: g 2669. DOI: 10.1136/bmj.g2669. 
24. Howie M.T., Sandblom G., Österberg J. 2017. The impact of pain frequency, pain localization and perceived cause of pain on quality of life after cholecystectomy. Scand. J. Gastroenterol. 52 (12): 1391-1397. doi: 10.1080 / 00365521.2017.1369564. Epub. 2017, Aug 28.

25. Kirk G., Kennedy R., McKie L., Diamond T., Clements B. 2011. Preoperative symptoms of irritable bowel syndrome predict poor outcome after laparoscopic cholecystectomy. Surg. Endosc. 25 (10): 3379-84. doi: 10.1007/s00464-011-1729-7. Epub. 2011, May 10.

26. Koishibayeva L.M., Turgunov Ye., Sandblom G., Teleuov M., Baimuratova M. 2017. Assessment of the quality of life of patients after cholecystectomy. Georgian Med. News. (270): 30-38.

27. Koishibayeva L.M., Sandblom G., Turgunov Y.M., Koishibayev Z.M. Quality-of-life assessment after cholecystectomy in a population-based cohort from Kazakhstan. Asian. J. Surg. 2020 Sep.; 43 (9): 948-949. doi: 10.1016/j.asjsur.2020.05.016. Epub 2020 Jun 9. PMID: 32532681.

28. Lamberts M.P., Den Oudsten B.L., Gerritsen J.J.G. M., Roukema J.A., Westert G.P., Drenth J.P.H., van Laarhoven C.J.H.M. 2015. Prospective multicentre cohort study of patient-reported outcomes after cholecystectomy for uncomplicated symptomatic cholecystolithiasis. Br. J. Surg. 102 (11): 1402-9. doi: 10.1002/bjs.9887. Epub. 2015, Jul 22.

29. Lamberts M.P., Kievit W., Gerritsen J.J.G.M., Roukema J.A., Westert G.P., Drenth J. P.H., van Laarhoven C.J.H.M. 2016. Episodic abdominal pain characteristics are not associated with clinically relevant improvement of health status after cholecystectomy. J. Gastrointest. Surg. 20 (7): 1350-8. doi: 10.1007/s11605-016-3156-5. Epub. 2016, May 17.

30. Lammert F., Gurusamy K., Ko C.W., Miquel J.F., Méndez-Sánchez N., Portincasa P., van Erpecum K.J., van Laarhoven C.J., Wang D.Q. Gallstones. Nat. Rev. Dis. Primers. 2016 Apr. 28; 2: 16024. doi: 10.1038/nrdp.2016.24. PMID: 27121416.

31. McLean K.A., Sheng Z., O'Neill S., Boyce K., Jones C., Wigmore S.J., Harrison E.M. 2017. The influence of clinical and patient-reported outcomes on post-surgery satisfaction in cholecystectomy patients. World J. Surg. 41 (7): 1752-1761. doi: 10.1007/s00268-017-3917-7.

32. Parkin E., Stott M., Brockbank J., Galloway S., Welch I., Macdonald A. 2017. Patientreported outcomes for acute gallstone pathology. World J. Surg. 41 (5): 1234-1238. doi: 10.1007/s00268016-3854-x.

33. Reibetanz J., Ickrath P., Hain J., Germer Ch.-T., Krajinovic K. 2013. Single-port laparoscopic cholecystectomy versus standard multiport laparoscopic cholecystectomy: a case-control study comparing the long-term quality of life and body image. Surg. Today. 43 (9): 1025-30. doi: 10.1007/s00595-0120393-4. Epub. 2012, Nov 2.

34. Russell M.L., Preshaw R.M., Brant R.F. 1996. Disease-specific quality of life: the Gallstone Impact Checklist. Clin. Invest. Med. 19 (6): 453-460.

35. Sandblom G., Videhult P., Karlson B.M., Wollert S., Ljungdahl M., Darkahi B., Liljeholm H., Rasmussen I.C. Validation of Gastrointestinal Quality of Life Index in Swedish for assessing the impact of gallstones on health-related quality of life. Value Health. 2009 Jan-Feb; 12 (1): 181-4. doi: 10.1111/j.1524-4733.2008.00396.x. PMID: 19911447.

36. Terwee C.B., Mokkink L.B., Knol D.L., Ostelo R.W., Bouter L.M., de Vet H.C. Rating the methodological quality in systematic reviews of studies on measurement properties: a scoring system for the COSMIN checklist. Qual. Life Res. 2012 May; 21 (4): 651-7. doi: 10.1007/s11136-011-9960-1. Epub 2011 Jul 6. PMID: 21732199; PMCID: PMC3323819.

37. Wanjura V., Lundstrom P., Osterberg J. 2014. Gastrointestinal quality-oflife after cholecystectomy: indication predicts gastrointestinal symptoms and abdominal pain. World J. Surg. 38: 3075-81.

38. Wanjura V., Sandblom G., Surg. World J. 2016. How do quality-of-life and gastrointestinal symptoms differ between post-cholecystectomy patients and the background population? 40 (1): 81-88. doi: 10.1007/s00268-015-3240-0.

39. Yu H., Ern-Hwei Chan E., Lingam P., Lee J., Wei Liang Woon W., Keem Low J., Shelat V. 2018. Index admission laparoscopic cholecystectomy for acute cholecystitis restores Gastrointestinal Quality of Life Index (GIQLI) score. Ann. Hepatobiliary Pancreat. Surg. 22 (1): 58-65. doi: 10.14701/ahbps.2018.22.1.58. Epub. 2018, Feb 26.

40. Zapf M., Denham W., Barrera E., Butt Z., Carbray J., Wang Ch., Linn J., Ujiki M. 2013 Patient-centered outcomes after laparoscopic cholecystectomy. Surg. Endosc. 27 (12): 4491-8. doi: 10.1007/s00464-013-3095-0. Epub. 2013, Aug 13. 


\section{ИНФОРМАЦИЯ ОБ АВТОРАХ}

Давыдкин Василий Иванович, кандидат медицинских наук, доцент, заведующий кафедрой госпитальной хирургии Национального исследовательского Мордовского государственного университета имени Н.П. Огарёва, г. Саранск, Россия

Федулов Руслан Игоревич, студент медицинского института Национального исследовательского Мордовского государственного университета имени Н.П. Огарёва, г. Саранск, Россия

Соболев Юрий Анатольевич, кандидат медицинских наук, доцент кафедры факультетской хирургии Оренбургского государственного медицинского

университета, г. Оренбург, Россия

Беляева Александра Игоревна, студентка лечебного факультета Оренбургского государственного медицинского

\section{INFORMATION ABOUT THE AUTHORS}

Vasily I. Davydkin, candidate of medical Sciences, associate Professor, head of the Department of hospital surgery with courses in traumatology and orthopedics, eye diseases, National research Mordovian state University, Saransk, Russia

Ruslan I. Fedulov, 6th-year student of the specialty «Medical science» of the National research Mordovian state University, Saransk, Russia

Yuri A. Sobolev, candidate of medical Sciences, associate Professor of the Department of faculty surgery of the Orenburg state medical University of the Ministry of Health of the Russian Federation, Orenburg, Russia

Alexandra I. Belyaeva, 6th year student of the faculty of medicine, Orenburg state medical University, Ministry of Health of the Russian Federation, Orenburg, Russia 